\title{
Integração de Modelos Econométrico e de Insumo-Produto para Previsões de Longo Prazo da Demanda de Energia no Brasil ${ }^{\star}$
}

\author{
- Rogério Silva de Mattos* \\ - Eduardo amaral haddad ***
}

\author{
- Fernando Salgueiro Perobelli $* \star$ \\ - WESLEM RODRIGUES FARIA $* \star \star \star$
}

\section{RESUMO}

Este trabalho apresenta um modelo tipo econométrico + insumo-produto para previsões de longo prazo do consumo de energia por setor de atividade no Brasil. São feitas previsões anuais para 2005-2010. A metodologia integra modelos econométricos de séries temporais com modelos de insumo-produto. Um resultado relevante obtido é a conexão entre modelos vetoriais auto-regressivos com ou sem mecanismos de correção de erros e modelos de insumo-produto fechados ou abertos. Dois cenários de previsão são considerados: um expansionista, com crescimento mais acelerado da economia; e um retraído, com crescimento amortecido. No caso mais provável do cenário expansionista, os resultados confirmam expectativas de que o estrangulamento energético ocorrerá a partir de 2009, antes do final da década. Direções para pesquisas futuras envolvendo o avanço da metodologia são consideradas ao final.

\section{Palavras-Chave}

modelo integrado, econométrico, insumo-produto, consumo de energia

\section{ABSTRACT}

The paper presents an econometric + input-output model for long-run forecasting of energy consumption by sector in Brazil. Yearly forecasts are produced for 2005-2010. The approach integrates a time series econometric model with an input-output model. A relevant result is a connection established between vector autoregressive models with or without error correction mechanisms and closed or open input-output models. Two forecasting scenarios are set-up: an expansionist scenario that predicts a faster economic growth; and a damped scenario that predicts a smoothed growth. In the more likely case of the expansionist scenario, expectations are confirmed that energy shortage will take place from 2009 on, thus before the end of the decade. Directions for further research on methodology improvements are considered at the end.

\section{KEYWORDS}

integrated model, econometric, input-output, energy consumption

JEL CLASSIFICATION

C 67, C32

+ Os autores agradecem ao Conselho Nacional de Desenvolvimento Científico e Tecnológico (CNPq) e ao Programa de Apoio a Grupos de Pesquisa da Universidade Federal de Juiz de Fora pelo apoio financeiro, e a Joaquim Guilhoto e Umberto Sesso pela cessão das matrizes de insumo-produto usadas neste trabalho.

* Doutor em Engenharia Elétrica - PUC-Rio e Professor do Mestrado em Economia Aplicada - FEA/UFJF. Endereço para contato: Universidade Federal de Juiz de Fora - Faculdade de Economia e Administração. CEP: 36036-330, Campus Universitário - Juiz de Fora - MG. E-mail: rogerio.mattos@ufjf.edu.br.

** Doutor em Economia - FEA/USP, Professor do Mestrado em Economia Aplicada - FEA/UFJF, Bolsista de Produtividade CNPq - Nivel II e Pesquisador - FAPEMIG. E-mail: fernando.perobelli@ufjf.edu.br.

$\star \star \star \quad P h . D$. em Economia - University of Illinois, Professor Titular do Departamento de Economia da FEA/USP, Pesquisador da Fundação Instituto de Pesquisas Econômicas - Fipe, Research Associate Professor do Regional Economics Applications Laboratory - REAL/UIUC, Coordenador do Núcleo de Economia Regional e Urbana da USP - Nereus e Bolsista do CNPq e da Fapesp. E-mail: ehaddad@usp.br.

$\star \star \star \star$ Mestrando em Economia-CEDEPLAR/UFMG. E-mail: weslemrodrigues@click21.com.br.

(Recebido em agosto de 2007. Aceito para publicação em julho de 2008). 


\section{$1 \quad$ INTRODUÇÃO}

Energia é um insumo essencial em economias industriais modernas. Conseqüentemente, sua disponibilidade tem papel proeminente para o desenvolvimento econômico de um país, estado ou região. No caso brasileiro, o suprimento adequado de energia é percebido atualmente como um dos principais condicionantes estratégicos para a retomada do crescimento econômico. Por exemplo, condições inadequadas de fornecimento de energia (disponibilidade insuficiente e/ou problemas de distribuição) podem implicar futuramente um aumento das importações de produtos energéticos, como petróleo e gás natural, vindo a deteriorar o saldo na balança comercial. Noutros casos, como o da energia elétrica, pode ocorrer a situação mais séria de interrupção do processo produtivo e, conseqüentemente, do próprio crescimento.

A crise de energia elétrica de 2001, que chegou a criar a necessidade de um programa de racionamento, fez aumentar as preocupaçóes da sociedade brasileira quanto a como garantir para o futuro o suprimento normal de energia. Diversas ações foram realizadas, como intensificação das pesquisas relacionadas ao aumento de eficiência técnica e ao uso de fontes alternativas de energia, ampliação dos programas e campanhas de conservação de energia, buscas de aperfeiçoamento do aparato regulatório (como o lançamento do novo modelo regulatório para o setor elétrico em 2004) e a elaboração de um plano decenal para ampliação da capacidade de geração de energia elétrica.

No entanto, uma importante dimensão do problema envolve a gestão estratégica do suprimento de energia a longo prazo. Essa gestão vem sendo coordenada pelos órgãos governamentais de planejamento energético e agências regulatórias em parceria com as empresas fornecedoras de energia. Para que tal gestão seja eficaz, necessário se faz o uso de instrumentos adequados para a geração de previsões a longo prazo da demanda de energia. Previsões agregadas sobre a demanda futura não são suficientes; é preciso traçar cenários de evolução futura que permitam visualizar em maior detalhe a estrutura de demanda de energia, por exemplo, desagregando as previsões segundo o tipo de energia, a categoria de uso final e as regiões espaciais de consumo.

Visando contribuir para o desenvolvimento de ferramentas de previsão da demanda de longo prazo de energia, este trabalho apresenta resultados de uma pesquisa voltada para a construção de um modelo integrado do tipo econométrico+insumoproduto $(\mathrm{EC}+\mathrm{IP})$ para a economia brasileira. Mais especificamente, será apresentada aqui uma versão de um modelo EC+IP voltado para se gerar previsões a longo prazo do consumo de energia por setor de atividade no Brasil. 
As motivações para este estudo se associam, de um lado, às necessidades mencionadas dessas previsões por parte dos agentes envolvidos com a gestão estratégica da energia, e, de outro, ao papel proeminente que modelos EC+IP vêm tendo na literatura recente de análise regional como uma ferramenta alternativa de previsões a longo prazo. Segundo Rey (2000) modelos integrados do tipo EC+IP podem ser uma alternativa superior a versóes individuais de modelos econométricos e de insumo-produto. Modelos EC+IP podem também funcionar de forma competitiva ou complementar com modelos de equilíbrio geral computável.

No que concerne ao módulo econométrico desses modelos, Rey (2000) também enfatiza a ausência na literatura sobre modelos EC+IP de esforços de modelagem coerentes com a moderna econometria de séries temporais. Tópicos como não-estacionariedade e co-integração têm sido muitas vezes ignorados pelos autores que trabalham com modelos EC+IP, que, alternativamente, vêm usando apenas métodos da econometria clássica. Na presente aplicação, apresenta-se uma integração de um modelo vetorial auto-regressivo (VAR) com um modelo de insumo-produto contendo um módulo de uso setorial de energia. Assim, além de prover previsões de consumo setorial para até o final desta década, este trabalho também explora as potencialidades de se integrar modelos VAR e de mecanismo de correção de erro (modelos VCE) com modelos de insumo-produto na construção de modelos EC+IP.

O trabalho está organizado da seguinte forma: na seção 2 , uma breve revisão da literatura sobre modelos EC+IP é feita; na seção 3, um modelo EC+IP para a economia brasileira, com um módulo de determinação do consumo setorial de energia, é apresentado em duas versões: um modelo fechado e um modelo aberto; na seção 4, os dados utilizados e respectivas fontes são descritos; na seção 5, os resultados da estimação do modelo VAR são apresentados juntamente com dois cenários alternativos e previsões condicionais a esses cenários; na seção 6, são apresentados os resultados do modelo EC+IP propriamente dito, em termos das previsões anuais do consumo setorial de energia para 2005-2010; na seção 7, uma breve discussão sobre as implicações dos resultados para a atual estratégia energética brasileira é feita; finalmente, na seção 8, são apresentadas as conclusões.

\section{LITERATURA SOBRE MODELOS EC+IP}

Considerando-se as últimas quatro décadas, o uso de modelos econométricos integrados com modelos de insumo-produto aparece de forma extensiva na literatura econômica. No entanto, a área de estudos regionais (regional science), que desde a 
década de 1950 vem usando diversas abordagens metodológicas (IZARD et al, 1960), tem sido a mais profícua em criar canais de síntese ou integração entre diversas formas de modelagem. Izard et al (1998), e também Rey, West e Janikas (2004) apontam a integração entre modelos EC e IP como o mais ativo e promissor desses canais de síntese atualmente.

Rey (2000) apresenta um survey da utilização de modelos EC+IP no contexto de análise regional e destaca duas fontes de motivação para a crescente utilização desse tipo de modelos integrados. Do ponto de vista teórico, a integração com modelos econométricos permite superar várias das notórias limitações ligadas à rigidez das hipóteses subjacentes aos modelos IP. Do ponto de vista prático, modelos integrados EC+IP têm alcançado, em vários casos, melhor performance preditiva, análises de impacto mais confiáveis e permitido tratamento da questão dos erros de medida. Mais recentemente, Rey, West e Janikas (2004) propõem métodos de simulação de Monte Carlo para se analisar a natureza da incerteza presente em modelos EC+IP.

Existem diferentes formas e estratégias pelas quais modelos EC podem ser integrados com modelos IP. Rey (2000) aponta basicamente três estratégias de integração: ligação (linking), determinação mútua (embedding) e acoplagem (coupling). $\mathrm{Na}$ estratégia de ligação, um dos módulos (EC ou IP) é exógeno ao outro, de forma que a interação entre eles é recursiva; nas estratégias de determinação mútua e de acoplagem, os módulos apresentam retroalimentação simultânea entre si, com o mecanismo de retroalimentação podendo ser completo (determinação mútua) ou parcial (acoplamento).

Israilevich, Mahidhara e Hewings (1994) discutem o papel do insumo-produto nos modelos integrados de EC+IP. Esses autores tentam captar qual a importância que a estrutura de insumo-produto (e.g tipo de agregação, método de construção e estimação dos coeficientes) tem sobre o resultado do modelo integrado. Le Sage e Rey (1994) discutem o uso de restrições presentes na integração dos modelos EC+IP, ou seja, como incorporar as informações do modelo de insumo-produto como restrições ao modelo econométrico. Os autores exploram no trabalho quais as implicações para os resultados de se tratar as restrições impostas pela estrutura de insumo-produto como estocásticas ou determinísticas.

Israilevich et al (1997) desenvolveram um modelo EC+IP (o Chicago Region Econometric Input-Output Model - CREIM) a fim de verificar como previsões ao nível macroeconômico regional influenciam o processo de mudança estrutural. A metodologia utilizada permite fazer previsões detalhadas de mudanças estruturais na economia de Chicago. O modelo deriva matrizes de insumo-produto para Chicago, no período 1975-2016, que identificam 36 setores industriais e três níveis de go- 
verno. Através dessas matrizes, é possível determinar qual o direcionamento das mudanças estruturais da economia da região metropolitana de Chicago.

No contexto brasileiro, os trabalhos com modelos EC+IP são ainda incipientes. Em um trabalho não publicado, Azzoni e Kadota desenvolvem um modelo EC+IP para a economia paulista, inspirando-se em modelos desenvolvidos pelo Regional Analysis Laboratory (REAL) da Universidade de Illinois que foram aplicados com sucesso nos EUA (CONWAY, 1990). Esses autores estimam equações econométricas para vários setores e de um modo que lhes permite incorporar a atualização dos coeficientes técnicos de IP em previsões setoriais de produção, emprego e renda no período 1994-2004. Mais recentemente, uma versão similar de modelo EC+IP foi usada pelo IPLANCE (2001) para analisar os impactos do racionamento de energia elétrica de 2001 sobre a economia cearense.

\section{MODELOS EC+IP PARA O BRASIL}

Os modelos EC+IP aqui desenvolvidos baseiam-se em versões simples para esses modelos discutidas em Rey, West e Janikas (2004). Duas versões de modelo EC+IP são exploradas: um modelo fechado e um modelo aberto. Os termos fechado e aberto dizem respeito à estruturação do módulo de insumo-produto (IP), que é uma das partes de um modelo EC+IP. No modelo fechado, um modelo VCE é integrado com um modelo de insumo-produto onde o consumo das unidades familiares é endogeneizado. No modelo aberto, por sua vez, um modelo VAR é integrado com um modelo de insumo-produto padrão, isto é, aberto em relação aos componentes da demanda final.

A construção dos modelos EC+IP apresentados nesta seção seguiu uma estratégia de integração por ligação (vide seção anterior) e uma abordagem de cima para baixo. Os componentes do modelo foram montados hierarquicamente para compor a estrutura global de cada versão (fechado ou aberto) de modelo EC+IP. A sequiência de montagem começa com o modelo econométrico usado para prever os itens de demanda final. Estes, por sua vez, são variáveis exógenas do modelo de insumo-produto. Assim, o próximo componente na hierarquia é o modelo de insumo-produto, que serve para prever os impactos sobre produção setorial. Isto, por sua vez, alimenta um componente de demanda setorial de energia que é adicionado. Para ambos os modelos EC+IP aqui apresentados, assumiu-se $n$ setores e apenas uma região (o Brasil).

Nesta seção, serão apresentados ambos os modelos, iniciando-se, porém, com um conjunto de identidades básicas que dá origem a ambas as versões de modelos EC+IP. Essas identidades são as seguintes: 


$$
\begin{aligned}
& Y_{t}=C_{t}+G_{t}+I_{t}+E_{X t} \\
& F_{t}=h_{C} C_{t}+h_{G} G_{t}+h_{I} I_{t}+n e_{t} \\
& n e_{t}=h_{E x} E_{X t}-h_{M} M_{t} \\
& X_{t}=A X_{t}+F_{t} \\
& E_{t}=P X_{t}
\end{aligned}
$$

onde $Y$ é a renda interna bruta, $C$ o consumo das famílias, $G$ os gastos do governo, $I$ o investimento privado, $E_{X}$ as exportações e $M$ as importações, todos medidos em valores monetários. $X, F$ e ne são, respectivamente, vetores $n \times 1$ de produção, demanda final e exportações líquidas por setor; por sua vez, $h_{C}, h_{G}, h_{I}, h_{E x}$ e $h_{M}$ são vetores $n \times 1$ de proporções de desagregação setorial dos respectivos componentes da demanda final, de tal forma que:

$$
\sum_{i=1}^{n} h_{C, i}=\sum_{i=1}^{n} h_{G, i}=\sum_{i=1}^{n} h_{I, i}=\sum_{i=1}^{n} h_{E x, i}=\sum_{i=1}^{n} h_{M}=1 .
$$

$P$ é uma matriz diagonal $n \times n$ cuja diagonal principal são coeficientes de uso setorial de energia medidos em tep/R $\${ }^{1}$ e $E$ é um vetor $n \times 1$ de consumo setorial de energia medido em tep. $\mathrm{O}$ subscrito $t$ indexa o tempo em termos anuais.

\subsection{Modelo VCE para a Demanda Final}

O modelo econométrico configura o primeiro módulo da hierarquia do modelo EC+IP e será aqui denominado módulo EC. Ele serve para caracterizar o processo gerador dos dados para os componentes da demanda final representados por $Y, C$, $G, I, E_{X}$ e $M$. Os componentes $Y$ e $C$ foram tratados como variáveis endógenas e os demais, $G, I, E_{X}$ e $M$, como exógenas. Note-se que todos os componentes da demanda final são, na verdade, variáveis exógenas para o módulo IP; porém, no âmbito do módulo EC as variáveis $C$ e $Y$ são tratadas como endógenas.

O objetivo em princípio seria estimar a relação entre $C$ e $Y$ segundo uma especificação keynesiana:

1 "Tep" é abreviatura de "toneladas de equivalente em petróleo" que serve como uma medida de conversão, para uma unidade de medida comum, das quantidades de diferentes fontes de energia. Isso permite que as quantidades de energia oriundas de fontes diferentes possam ser adicionadas. 


$$
C_{t}=c_{0}+c_{1} Y_{t}+\varepsilon_{t}
$$

e considerando-se também a identidade contábil para $Y_{t}$ representada pela equação (1). No caso da equação (6), é fato bem conhecido que não se deve estimar diretamente a relação entre $C$ e $Y$ com essas variáveis em níveis, sobretudo se elas forem co-integradas (e.g., ENDERS, 2003). Problemas de estimação e uso ineficiente de informações sobre o processo gerador dos dados podem acontecer, devendo-se preferir especificar modelos para as variáveis em diferenças estacionárias. Isto implica representar a princípio a relação entre $C$ e $Y$ como um modelo VCE:

$$
\Delta W_{t}=-\alpha\left(C_{t-1}-c_{0}-c_{1} Y_{t-1}\right)+\Theta_{1} \Delta W_{t-1}+\Psi_{0} \Delta Z_{t}+\Psi_{1} \Delta Z_{t-1}+e_{t}
$$

onde: $W_{t}=\left[\begin{array}{c}C_{t} \\ Y_{t}\end{array}\right], \quad Z_{t}=$ vetor $(m \times 1)$ de variáveis exógenas, $\alpha=\left[\begin{array}{l}\alpha_{1} \\ \alpha_{2}\end{array}\right]$,

$$
\begin{aligned}
\Theta_{1} & =\left[\begin{array}{ll}
\theta_{11} & \theta_{12} \\
\theta_{21} & \theta_{22}
\end{array}\right] \\
\Psi_{0} & =\left[\begin{array}{lll}
\gamma_{0,11} & \cdots & \gamma_{0,1 m} \\
\gamma_{0,21} & \cdots & \gamma_{0,2 m}
\end{array}\right], \Psi_{1}=\left[\begin{array}{lll}
\gamma_{1,11} & \cdots & \gamma_{1,1 m} \\
\gamma_{1,21} & \cdots & \gamma_{1,2 m}
\end{array}\right] \text { e } e_{t}=\left[\begin{array}{l}
e_{1 t} \\
e_{2 t}
\end{array}\right] .
\end{aligned}
$$

Note-se que o vetor $W$ contém as variáveis endógenas do modelo VCE. Está implícito na representação em (7) que todas as variáveis em níveis são integradas de ordem 1 , isto é, são $I(\mathrm{l})$, de modo que suas primeiras diferenças são $I(0)$. Admite-se que as variáveis exógenas possam ter efeitos contemporâneos e que a defasagem máxima do modelo VCE seja a priori de um ano. A última restrição baseia-se no fato de que os dados usados para estimação do modelo VCE são anuais. De qualquer forma, procedimentos estatísticos apropriados foram usados para ajudar na identificação de uma defasagem máxima (ver seção 5 ).

Deve ser observado, porém, que o conjunto de variáveis exógenas representado por $Z$ pode incluir diversas variáveis e não apenas aquelas consideradas aqui, isto é, $G, I$, $E_{X}$ e $M$. Além disso, neste trabalho, o vetor $Z$ não foi considerado explicitamente no modelo econométrico estimado, e que está apresentado adiante na seção 5 , porque 
as variáveis exógenas consideradas aparecem embutidas nas identidades contábeis (l) e (3), isto é, para a renda e para as exportações líquidas, respectivamente.

O mecanismo de correção de erros em (7) é representado pelo termo $-\alpha\left(C_{t}-c_{0}-c_{1} Y_{t}\right)$ e tem o papel de captar os efeitos que desvios na relação de longo prazo entre $C$ e $Y$ produzem sobre a dinâmica de curto prazo para essas variáveis. $\mathrm{O}$ vetor $\alpha$ contém os coeficientes de ajustamento de cada variável endógena aos desvios na relação de longo prazo. Seu uso com o sinal negativo em (7) foi feito aqui apenas por conveniência. Note-se que, no caso de $\alpha=0$ ou de $C$ e $Y$ não serem cointegradas, este termo não deve estar presente no modelo econométrico em (7), que neste caso se reduziria a um modelo VAR com variáveis exógenas contemporâneas (ENDERS, 2003, p. 338).

Identificaram-se duas formas pelas quais o modelo econométrico em (7) pode ser integrado com o módulo IP. Na primeira, desde que $C$ e $Y$ sejam co-integradas, obtémse um modelo IP fechado onde o consumo das unidades familiares é endogeneizado. Na segunda, se $C$ e $Y$ não forem co-integradas, obtém-se uma representação padrão de modelo IP aberto. Esses aspectos são mostrados em detalhe nas duas próximas sub-seções, porém antes será apresentado um resultado relevante que será usado, que corresponde à representação da equação de consumo com esta variável em níveis e que está implícita no modelo VCE em (7).

Assim, escrevendo-se apenas a equação para $\Delta C$ em (7), obtém-se:

$$
\Delta C_{t}=-\alpha_{1}\left(C_{t-1}-c_{0}-c_{1} Y_{t-1}\right)+\left[\begin{array}{ll}
\theta_{11} & \theta_{12}
\end{array}\right] \Delta W_{t-1}+\sum_{i=0}^{1}\left[\begin{array}{lll}
\gamma_{i, 11} & \gamma_{i, 12} & \gamma_{i, 13}
\end{array}\right] \Delta Z_{t-i}+e_{1 t}
$$

Definindo-se, para fins de simplificação,

$$
V_{t}=\left[\begin{array}{ll}
\theta_{11} & \theta_{12}
\end{array}\right] \Delta W_{t-1}+\sum_{i=0}^{1}\left[\begin{array}{lll}
\gamma_{i, 11} & \gamma_{i, 12} & \gamma_{i, 13}
\end{array}\right] \Delta Z_{t-i}+e_{1 t}
$$

então:

$$
\Delta C_{t}=-\alpha_{1}\left(C_{t-1}-c_{0}-c_{1} Y_{t-1}\right)+V_{t}
$$

ou:

$$
C_{t}=C_{t-1}-\alpha_{1}\left(C_{t-1}-c_{0}-c_{1} Y_{t-1}\right)+V_{t}
$$


Agora, considerando que $Y_{t-1}=Y_{t}-\Delta Y_{t}$ e realizando algumas manipulações algébricas:

$$
C_{t}=C_{t-1}-\alpha_{1}\left(C_{t-1}-c_{0}+c_{1} \Delta Y_{t}\right)+\alpha_{1} c_{1} Y_{t}+V_{t}
$$

Como $Y_{t}$ é a soma dos valores adicionados em todos os setores da economia, ele pode ser representado como $Y_{t}=i_{n}^{\prime} Q X_{t}$, onde $i_{n}$ é um vetor unitário $n \times 1$ e $Q$ é uma matriz diagonal $n \times n$ de coeficientes de valor adicionado por setor. Assim, substituindo-se $Y_{t}$ por $i_{n}^{\prime} Q X_{t}$ na equação acima, obtém-se a seguinte versão para o consumo contemporâneo em níveis:

$$
C_{t}=C_{t-1}-\alpha_{1}\left(C_{t-1}-c_{0}+c_{1} \Delta Y_{t}\right)+\alpha_{1} c_{1} i_{n}^{\prime} Q X_{t}+V_{t}
$$

A equação (9) será usada a seguir no desenvolvimento da integração do modelo VCE com o modelo IP.

\subsection{Modelo IP Fechado}

O modelo IP é o segundo componente da hierarquia do modelo EC+IP. Dadas as informações numéricas para os componentes da demanda final, é possível usar o módulo IP para se computar vetores de impacto sobre produção e demanda de energia por setor de atividade. Manipulando as expressões (1) a (5), é possível desenvolver duas versões: um modelo IP aberto ou um modelo IP fechado.

O modelo fechado é baseado na endogeneização do consumo das unidades familiares, o que permite captar efeitos de retroalimentação desse consumo sobre o restante da economia. O modelo fechado é desenvolvido a partir, inicialmente, da substituição de (9) em (2):

$$
F_{t}=h_{C}\left[C_{t-1}-\alpha_{1}\left(C_{t-1}-c_{0}+c_{1} \Delta Y_{t}\right)+\alpha_{1} c_{1} i_{n}^{\prime} Q X_{t}+V_{t}\right]+h_{G} G_{t}+h_{I} I_{t}+n e_{t}
$$

de onde é imediato que:

$$
F_{t}-h_{C} \alpha_{1} c_{1} i_{n}^{\prime} Q X_{t}=h_{C}\left[C_{t-1}-\alpha_{1}\left(C_{t-1}-c_{0}+c_{1} \Delta Y_{t}\right)+V_{t}\right]+h_{G} G_{t}+h_{I} I_{t}+n e_{t}
$$

Usando este resultado e definindo $F_{t}^{*}=F_{t}-h_{C} \alpha_{1} c_{1} i_{n}^{\prime} Q X_{t}$, pode-se reescrever a equação de IP em (4) como: 


$$
X_{t}=A X_{t}+h_{C} \alpha_{1} c_{1} i_{n}^{\prime} Q X_{t}+F_{t}^{*}
$$

Segue, após manipulações algébricas simples, que:

$$
\begin{aligned}
& X_{t}=\Gamma^{-1} F_{t}^{*} \\
& \Gamma=\left[I-A-h_{C} \alpha_{1} c_{1} i_{n}^{\prime} Q\right] \\
& F_{t}^{*}=h_{C}\left[C_{t-1}-\alpha_{1}\left(C_{t-1}-c_{0}+c_{1} \Delta Y_{t}\right)+V_{t}\right]+h_{G} G_{t}+h_{I} I_{t}+n e_{t}
\end{aligned}
$$

As expressões (10)-(12) são a base para se fazer as previsões de impacto sobre produção por setor de atividade com o modelo fechado. Note-se que as variáveis exógenas são apenas $G, I, E_{X} e M$ (as duas últimas dão origem à ne segundo (3)). O cômputo da demanda de energia por setor é feito com a equação (5).

A forma de endogeneizar o consumo usada acima baseia-se nas informações do modelo econométrico. Segundo Rey (2000) essa forma tem sido preferida por vários autores, embora os mesmos não tenham, em geral, seguido a econometria de séries temporais como feito aqui. Deve ser observado que endogeneizar o consumo a partir do modelo econométrico é diferente do procedimento usual de se fechar um modelo IP para as unidades familiares inserindo colunas e linhas adicionais na matriz $A$ (e.g., MILLER; BLAIR, 1985).

\subsection{Modelo IP Aberto}

O modelo IP aberto é desenvolvido de forma análoga, mas sem se considerar efeitos de retroalimentação do consumo familiar sobre o restante da economia. Parte-se diretamente da equação (7) e, após manipulação algébrica, obtém-se:

$$
\begin{aligned}
& X_{t}=\Gamma^{-1} F_{t} \\
& \Gamma=[I-A] \\
& F_{t}=h_{C} C_{t}+h_{G} G_{t}+h_{I} I_{t}+n e_{t}
\end{aligned}
$$

As expressões (13)-(15) formam o modelo IP aberto. Note-se que, neste caso, as variáveis exógenas são $C, G, I, E_{X} e M$; a variável $C$ tem de ser projetada pelo modelo 
VAR (equação (7) sem o termo de co-integração), ao passo que para as outras são especificados cenários futuros. O cômputo da demanda de energia por setor é feito com a equação (5).

\subsection{Consistência entre Modelos EC+IP Fechado e Aberto}

É interessante notar que os conjuntos de expressões (10)-(12) e (13)-(15) são consistentes entre si. Se $\alpha_{1}=0$, o modelo fechado transforma-se automaticamente no modelo aberto $\operatorname{com} F^{*}=F$. Isso estabelece uma conexão dentro do modelo EC+IP com a existência ou não de co-integração entre $C$ e $Y$. Se $C$ e $Y$ não forem co-integradas, então o termo de correção de erro no modelo VCE em (7) não existe e a forma apropriada, neste caso, para se representar o relacionamento dinâmico entre $C$ e $Y$ seria um modelo VAR para as primeiras diferenças dessas variáveis. Mais ainda, o modelo IP tem de ser o modelo aberto em (13)-(15).

Se for o contrário, isto é, se $\alpha_{1} \neq 0$, então existe um mecanismo de correção de erros e $C$ e $Y$ são co-integradas. Neste caso, o modelo VCE é o apropriado e, além disso, o modelo IP tem de ser o modelo fechado. Esses fatos podem ser resumidos como segue:

a) Se $C$ e $Y$ forem co-integradas, o modelo EC+IP tem de ser representado na parte EC por um modelo VCE e na parte IP por um modelo IP fechado em relação ao consumo das unidades familiares;

b) Se $C$ e $Y$ não forem co-integradas, o modelo EC+IP tem de ser representado na parte EC por um modelo VAR para $\Delta C$ e $\Delta Y$ e na parte IP por um modelo IP aberto.

\subsection{Previsões e Cenários}

O modelo EC+IP desenvolvido acima apresenta, portanto, uma versão fechada e outra aberta. Ambas as versões podem ser usadas para se fazer as previsões futuras de longo prazo para o consumo setorial de energia. Os resultados teóricos apresentados na seção anterior, no entanto, implicam que, no caso específico do modelo EC+IP aqui construído, o processo de estimação empírica do modelo econométrico tem um papel importante, pois os testes de co-integração irão determinar, também, qual versão do modelo EC+IP deverá ser usada para se gerar as previsões.

Em ambas as versões, as variáveis exógenas do modelo integrado são $G, I, E_{X}$ e $M$. Portanto, a realização de previsões com o modelo EC+IP para anos futuros depen- 
de de previsões externas para essas variáveis exógenas. Optou-se aqui por trabalhar com cenários futuros para essas variáveis. Tal esforço mostra-se mais produtivo no presente contexto, pois diferentes condições para a evolução da economia e seus impactos sobre a demanda setorial de energia podem ser medidos e analisados. Os cenários construídos para as variáveis exógenas no período de análise, isto é, 2005 a 2010 são descritos na seção 5 juntamente com os resultados da construção do modelo econométrico.

\section{BASE DE DADOS}

Esta seção descreve os dados utilizados na construção do modelo EC+IP. Para a estimação do modelo econométrico no módulo EC, foram usadas as séries históricas anuais, de 1960 a 2004, produzidas pelo Sistema de Contas Nacionais do IBGE para os componentes da demanda final: PIB $(Y)$, consumo das famílias $(C)$, investimento $(I)$, exportações $\left(E_{X}\right)$ e importações $(M)$. As séries foram obtidas no site do Ipeadata. A série para os gastos do governo $(G)$ foi obtida por resíduo usando-se a equação (1). No caso de $Y, C, G$ e $I$, as séries foram obtidas a preços correntes em $\mathrm{R} \$$ mil e convertidas a preços constantes em R $\$$ mil de 2004 usando-se o deflator implícito do PIB. As séries de exportações e importações foram obtidas em US\$ mil e convertidas para preços correntes em $\mathrm{R} \$$ mil usando-se a taxa de câmbio média anual $\mathrm{R} \$$ / US\$ antes de serem convertidas a preços constantes em R \$ mil de 2004.

Para a construção do módulo IP, foram usadas as matrizes de IP estimadas de 1997 a 2001 por Guilhoto e Sesso Filho (2005) e os dados de uso de energia em tep disponíveis para o ano de 2003 no Balanço Energético Nacional (BEN) de 2004 (Brasil/MME, 2004). Foi necessária uma compatibilização entre essas duas fontes de dados porque as matrizes de IP foram construídas para 42 setores e os dados do BEN só estavam disponíveis para 13 setores. Assim, seguiu-se um procedimento de agregação dos setores das matrizes de IP para se compatibilizar com os 13 setores do BEN. Vale observar, porém, que essa compatibilização não é completa porque exclui o segmento de consumo residencial que é parte integrante do consumo final energético reportado no BEN.

Os dados das matrizes de IP permitiram a construção da matriz de coeficientes técnicos $A$, os vetores de desagregação $h_{C}, h_{G}, h_{I}, h_{E x}$ e $h_{M}$, e a matriz de coeficientes de valor adicionado $Q$. As matrizes $A$ e $Q$ foram construídas usando-se apenas o ano de 2001, que retrata as informações mais atualizadas disponíveis, mas os vetores de desagregação foram computados como médias de 1997-2001. Os dados de 2003 do BEN permitiram a construção da matriz de coeficientes de energia $P$. 


\section{RESULTADOS PARA O MODELO ECONOMÉTRICO}

Esta seção apresenta a estimação do modelo econométrico que compõe o modelo EC+IP e que foi especificado na equação (7). O modelo econométrico representa o processo gerador dos dados para os componentes da demanda final dentro da restrição de que $C$ e $Y$ são endógenas e $G, I, E_{X}$ e $M$ são exógenas. Os procedimentos de estimação seguiram os passos usuais da econometria de séries temporais. Primeiro, foi analisada a situação de não-estacionariedade das séries com testes de raízes unitárias; em seguida, foi verificada a existência ou não de co-integração entre as variáveis e, finalmente, foi realizada a estimação do modelo econométrico propriamente dito. Ao final da seção, são apresentadas também previsões condicionais a cenários para as variáveis exógenas usando-se o modelo estimado.

As cinco variáveis $C, Y, G, I$ e $N E$ foram submetidas ao teste de Dickey e Fuller Aumentado (ADF) para raízes unitárias. Os resultados estão apresentados na Tabela 1. Para cada variável testaram-se três situações:

a) série em nível com intercepto;

b) série em nível com intercepto e tendência e

c) série em primeiras diferenças.

O número de defasagens em cada caso foi determinado usando-se os critérios de informação de Akaike (AIC) e Schwarz (SIC). Como reportado na Tabela 1, todas as variáveis em níveis mostraram-se não-estacionárias sob as duas situações a) e b). A estacionariedade de cada série só foi obtida aplicando-se a primeira diferença, de forma que todas se mostraram integradas de ordem 1 ou $I(\mathrm{l})$. 


\section{TABELA I - TESTES DE RAÍZES UNITÁRIAS (ADF)}

\begin{tabular}{|c|c|c|c|c|c|c|}
\hline \multirow{2}{*}{ Variável } & \multirow{2}{*}{ Característica } & \multirow{2}{*}{$\mathrm{n}^{0}$ de lags } & \multirow{2}{*}{ razão $t$} & \multicolumn{3}{|c|}{ Valor crítico } \\
\hline & & & & $1 \%$ & $5 \%$ & $10 \%$ \\
\hline$\overline{C_{t}}$ & intercepto & 0 & $-2,385$ & $-3,633$ & $-2,948$ & $-2,613$ \\
\hline$C_{t}$ & intercepto e tendência & 0 & $-2,295$ & $-4,244$ & $-3,544$ & $-3,205$ \\
\hline$\Delta C_{t}$ & intercepto & 0 & $-5,022^{*}$ & $-3,633$ & $-2,948$ & $-2,613$ \\
\hline$Y_{t}$ & intercepto & 0 & $-1,331$ & $-3,633$ & $-2,948$ & $-2,613$ \\
\hline$Y_{t}$ & intercepto e tendência & 0 & $-2,484$ & $-4,244$ & $-3,544$ & $-3,205$ \\
\hline$\Delta Y_{t}$ & intercepto & 0 & $-4,702^{*}$ & $-3,633$ & $-2,948$ & $-2,613$ \\
\hline$G_{t}$ & intercepto & 0 & $-1,401$ & $-3,633$ & $-2,948$ & $-2,613$ \\
\hline$G_{t}$ & intercepto e tendência & 0 & $-3,309$ & $-4,244$ & $-3,544$ & $-3,205$ \\
\hline$\Delta G_{t}$ & intercepto & 1 & $-6,210^{*}$ & $-3,633$ & $-2,948$ & $-2,613$ \\
\hline$l_{t}$ & intercepto & 0 & $-2,124$ & $-3,633$ & $-2,948$ & $-2,613$ \\
\hline$I_{t}$ & intercepto e tendência & 0 & $-3,495$ & $-4,244$ & $-3,544$ & $-3,205$ \\
\hline$\Delta I_{t}$ & intercepto & 0 & $-5,950^{*}$ & $-3,633$ & $-2,948$ & $-2,613$ \\
\hline$N E_{t}$ & intercepto & 1 & $-1,753$ & $-3,633$ & $-2,948$ & $-2,613$ \\
\hline$N E_{t}$ & intercepto e tendência & 4 & $-2,842$ & $-4,244$ & $-3,544$ & $-3,205$ \\
\hline$\Delta N E_{t}$ & intercepto & 0 & $-4,380^{*}$ & $-3,633$ & $-2,948$ & $-2,613$ \\
\hline
\end{tabular}

Fonte: Elaboração dos autores com dados descritos na seção 4.

* Indica rejeição da hipótese nula de não-estacionariedade a $1 \%$ de significância.

A Tabela 2 apresenta testes de co-integração entre as variáveis endógenas $C$ e $Y$. Foram usados os testes de Johansen e de Engle e Granger. O segundo é baseado na aplicação do teste $\mathrm{ADF}$ aos resíduos da regressão em níveis entre $C$ e $Y$. Ambos os testes rejeitaram, com $5 \%$ de significância, a hipótese nula de co-integração. Este resultado é um tanto inesperado na medida em que se espera que consumo e renda sejam co-integrados. Várias hipóteses podem ser levantadas para esse resultado. Em primeiro lugar, estaria o longo período (44 anos) considerado na análise, ao longo do qual provavelmente ocorreram mudanças de diversas naturezas (tecnológicas, de padrão e preferências de consumo etc.) que impactaram no relacionamento de longo prazo entre $C$ e $Y$. O Gráfico 1 mostra a evolução da razão $C / Y$ ao longo do período de dados (entre 1960 e 2004) e é bastante sugestivo de um decréscimo latente nesta razão. Em segundo lugar, o mesmo Gráfico 1 evidencia um descolamento entre $C$ e $Y$ acontecendo mais recentemente, a partir de 1997, período que coincide com uma dificuldade cada vez maior de se recuperar o emprego formal com crescimento do PIB. Outras hipóteses poderiam ser ainda apontadas, mas foge ao escopo deste trabalho ir mais a fundo nessa questão. ${ }^{2}$

2 A ausência de co-integração entre $C$ e $Y$ foi detectada mesmo quando se realizou um teste tipo Engle e Granger (não reportado) com incorporação das variáveis exógenas $G, I$ e $N E$ na equação de cointegração. Entretanto, cabe ressaltar que no presente trabalho a relação entre $C$ e $Y$ está sendo modelada na tradição keynesiana, onde o consumo depende exclusivamente da renda. Autores como Fisher, Modigliani e Friedman incorporaram questôes como renda futura, escolha intertemporal, papel da 


\section{TABELA 2 - TESTES DE CO-INTEGR AÇÃO ENTRE CONSUMO E RENDA}

\section{Johansen}

Hipótese do teste: constante na equação de co-integração

\begin{tabular}{|c|c|c|c|c|}
\hline \multirow{2}{*}{ Autovalor } & \multirow{2}{*}{ Razão de Verossimilhança } & \multicolumn{2}{|c|}{ Valores críticos } & \multirow{2}{*}{$\begin{array}{c}\text { № de } \operatorname{ECs}^{*} \text { na Hip } \\
\text { Nula }\end{array}$} \\
\hline & & $5 \%$ & $1 \%$ & \\
\hline 0,205 & 9,199 & 15,41 & 20,04 & 0 \\
\hline 0,033 & 1,167 & 3,76 & 6,65 & máx. 1 \\
\hline
\end{tabular}

\section{Engle-Granger}

Regressão em níveis: $C=1,33 \times 10^{8}+0,514$ Y

Teste ADF sobre resíduos de regressão

\begin{tabular}{ccccc}
\hline \multirow{2}{*}{$n^{0}$ de lags } & \multirow{2}{*}{ razão t } & \multicolumn{3}{c}{ valor crítico } \\
\cline { 3 - 5 } & & $1 \%$ & $5 \%$ & $10 \%$ \\
\hline 1 & $-2,477$ & $-3,642$ & $-2,953$ & $-2,615$ \\
\hline
\end{tabular}

Fonte: Elaboração dos autores com dados descritos na seção 4 .

Nota: ${ }^{*} E C=$ equação de co-integração.

\section{GRÁFICO I - EVOLUÇÃO DA RAZÃO C/Y}

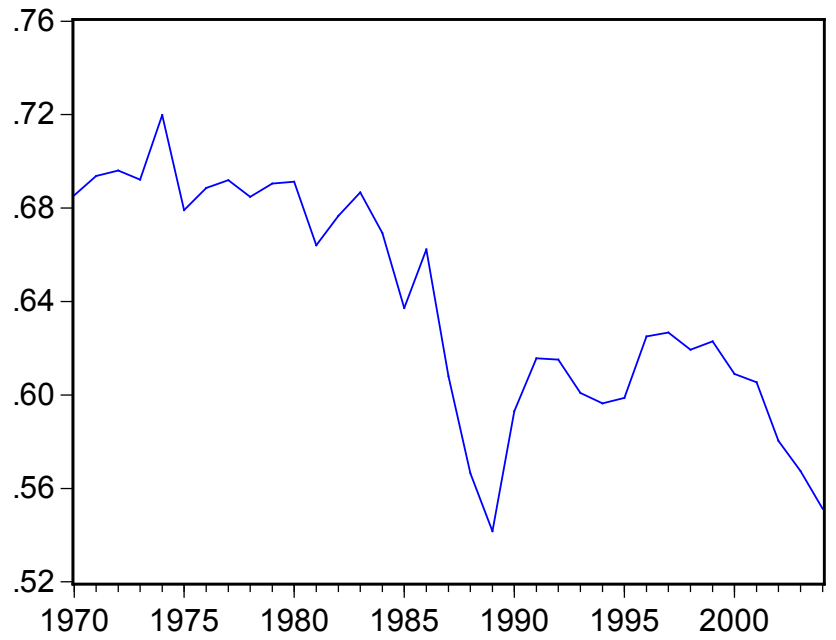

Fonte: Elaboração dos autores com dados descritos na seção 4.

Diante da ausência de co-integração entre $C$ e $Y$ detectada pelos testes estatísticos, o procedimento apropriado envolve estimar o modelo econométrico como um modelo VAR e não do tipo VCE. Assim, estimou-se um modelo VAR e os resultados estão apresentados na Tabela 3. A defasagem máxima usada no VAR foi de um ano,

riqueza, renda permanente, dentre outras. Logo, ao utilizar somente $C$ e $Y$ para especificar a relação de consumo é admissível que as duas variáveis não co-integrem. 
coincidindo com a minimização dos critérios AIC e SIC. Impôs-se a restrição de coeficiente nulo para $\Delta C_{t-1}$ porque esta variável apresentava forte correlação com $\Delta \Upsilon_{t-1}$. Na equação de consumo, foram incluídas três variáveis dummy: D81, D87 e D88, do tipo impulso (valem 1, respectivamente, em 1981, 1987 e 1988 e 0 nos demais anos), para captar uma redução mais abrupta do consumo que ocorreu nos anos de 1981, 1987 e 1988. O ano de 1981 marca o início de uma política recessiva, ao passo que os anos de 1987 e 1988 coincidem com a fase após a expansão de consumo ocorrida no Plano Cruzado em 1986 e também com a contração da renda promovida pelo Plano Bresser. Na equação de renda, foram incluídas apenas duas variáveis dummy: D81 e D90, e a segunda capta os efeitos recessivos da hiperinflação do final da década de 80 e do choque recessivo decorrente do Plano Collor.

Foram configurados dois cenários futuros alternativos para as variáveis exógenas, tal como apresentado na Tabela 4 . A partir desses cenários, foram feitas previsões anuais para 2005-2010 usando-se o modelo econométrico (VAR) apresentado na Tabela 3. Observe-se, porém, que esse modelo não incorpora explicitamente as variáveis exógenas $G, I$, e $E x$. No entanto, elas fazem parte da determinação de $Y$ pela identidade contábil (1). Assim, no cômputo das previsões para as variáveis endógenas foi usada apenas a equação para a variação do consumo da Tabela 3 porque as previsóes para as variações da renda foram feitas segundo:

$$
\Delta Y_{t}=\Delta C_{t}+\Delta G_{t}+\Delta I_{t}+\Delta E_{X t}
$$

que é a versão em primeiras diferenças da equação 1 .

Os cenários foram construídos da forma usual feita na literatura, isto é, para representar situações antagônicas. Assim, o primeiro cenário, denominado cenário expansionista, retrata uma situação de maior dinamismo da economia brasileira, com todas as variáveis exógenas se expandindo a partir de 2006 até 2010, da seguinte forma:

- Gastos do governo: crescimento de 5\% ao ano;

- Investimentos: crescimento de 5\% ao ano;

- Exportaçóes: crescimentos de $4 \%$ em 2006, 3\% ao ano em 2007-2008 e 2\% ao ano em 2009-2010;

- Importações: crescimentos de 4,5\% em 2006, 3,5\% ao ano em 2007-2008 e $2,5 \%$ ao ano em 2009-2010;

O outro cenário, denominado cenário retraído, retrata uma situação mais moderada, com expansão amortecida das variáveis exógenas, da seguinte forma: 
- Gastos do governo: crescimento de 5\% ao ano em 2006-2007 e 0\% ao ano em 2008-2010;

- Investimentos: decréscimo de -5\% ao ano em 2006-2007 e 0\% ao ano em 20082010;

- Exportaçóes: crescimentos de 4\% em 2006, 2\% em 2007 e 0\% ao ano em 20082010;

- Importações: crescimentos de 5\% em 2006, 2,5\% em 2007 e $0 \%$ ao ano em 2008-2010;

Em ambos os cenários, o ano de 2005 é configurado da mesma maneira e reflete previsões para esse ano estabelecidas pelos autores deste trabalho, mas que foram subsidiadas por previsões macroeconômicas elaboradas por institutos especializados como Project Link Research Centre (10/06/2005).

O resultado da aplicação do modelo econométrico da Tabela 3 em cada um desses cenários são as previsões para as variáveis endógenas $C$ e $Y$ no período 2005-2010 apresentadas na Tabela 4. No cenário expansionista, o consumo sai de cerca de R \$ 1,03 trilhões em 2005 e cresce suavemente até atingir R \$ 1,1 trilhões em 2010, um acréscimo de $6,8 \%$ ao longo dos 5 anos. No mesmo cenário, a renda evolui de R \$ 1,8 trilhões em 2005 para R \$ 2,1 trilhões em 2010, um acréscimo de 16\% no mesmo período. No cenário retraído, tanto o consumo quanto a renda ficam praticamente estagnados. 
TABELA 3 - MODELO VAR COM RESTRIÇÕES

\begin{tabular}{lcc}
\hline Variáveis & $\Delta C_{\mathrm{t}}$ & $\Delta Y_{\mathrm{t}}$ \\
\hline$\Delta C_{\mathrm{t}-1}$ & - & - \\
& - & - \\
$\Delta Y_{\mathrm{t}-1}$ & 0.241444 & 0.303703 \\
& 1.962320 & 2.073643 \\
Constante & 35142563 & 32952667 \\
& 3.247981 & 4.437609 \\
$\mathrm{D} 81_{\mathrm{t}}$ & $-1.05 \mathrm{E}+08$ & $-1.06 \mathrm{E}+08$ \\
& -4.201734 & -3.365523 \\
$\mathrm{D} 87_{\mathrm{t}}$ & -79463585 & - \\
& -3.192540 & - \\
$\mathrm{D} 88_{\mathrm{t}}$ & -81032738 & - \\
& -3.360653 & - \\
$\mathrm{D} 90_{\mathrm{t}}$ & - & $-1.02 \mathrm{E}+08$ \\
& - & -3.355900 \\
$T$ & -964539.8 & - \\
& -2.153852 & - \\
\hline $\mathrm{R}^{2}$ & 0.593742 & 0.444972 \\
$\mathrm{R}^{2}$-ajustado & 0.518509 & 0.387556 \\
$\mathrm{AIC}$ & 36.96204 & 37.37586 \\
$\mathrm{SC}$ & 37.23414 & 37.55725 \\
\hline
\end{tabular}

Fonte: Elaboração dos autores com dados descritos na seção 4.

Obs: Período de estimação: 1962-2004. Observações usadas: 43. Estatísticas $t$ entre parênteses. 


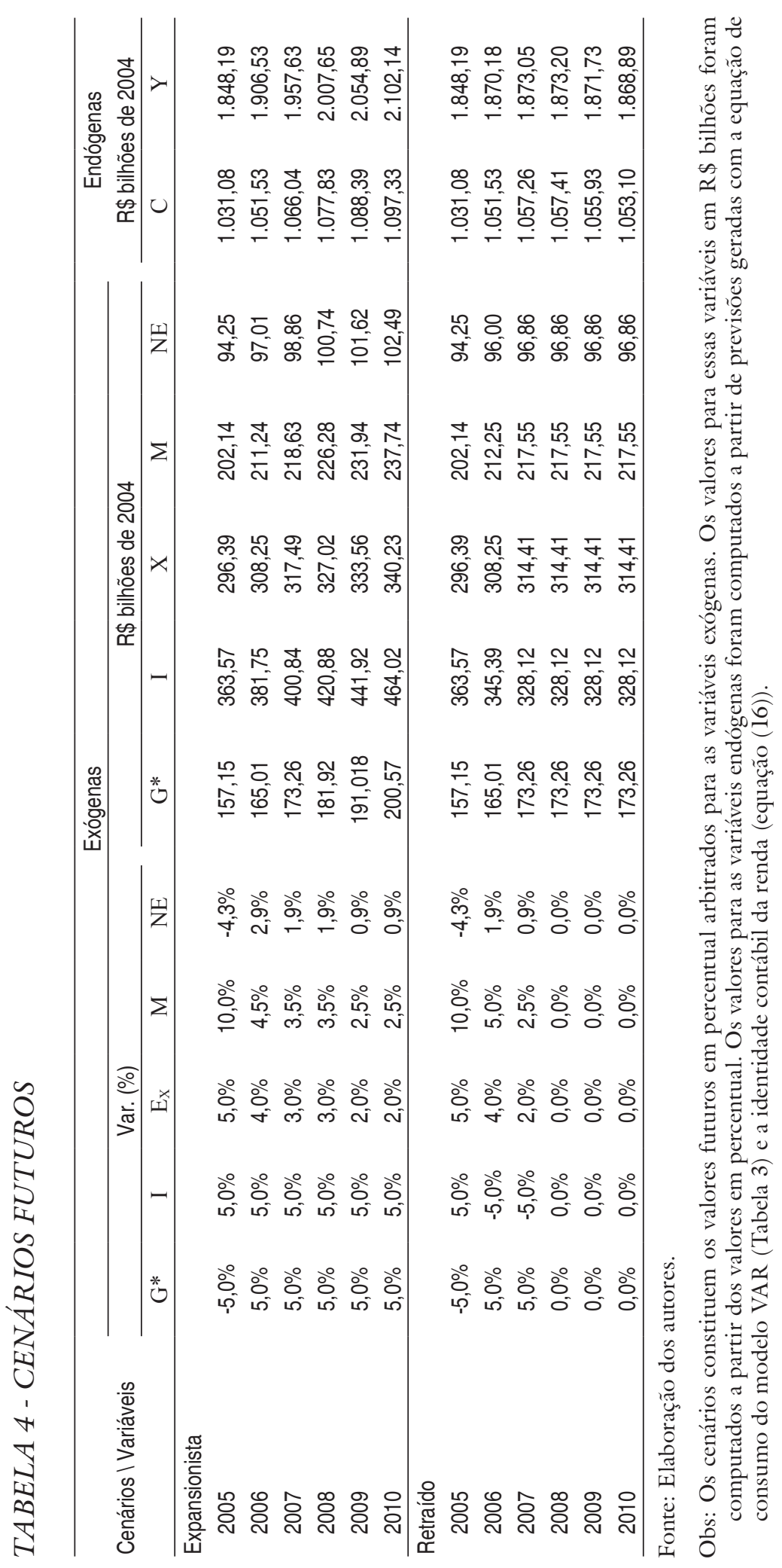




\section{RESULTADOS PARA O MÓDULO DE ENERGIA}

Os dois cenários para as variáveis exógenas e as respectivas previsões, feitas com o modelo VAR, para as variáveis endógenas (duas últimas colunas da Tabela 4) foram usados para alimentar o módulo IP do modelo EC+IP. Devido à ausência detectada de co-integração entre $C$ e $Y$, a versão do modelo IP usada para as previsões setoriais de uso de energia foi o modelo aberto (ver seção 3). A partir disso, foram computadas as previsões anuais de consumo de energia por setor para o mesmo período 2005-2010. Os resultados para o cenário expandido estão apresentados na Tabela $5 \mathrm{e}$ os correspondentes para o cenário retraído na Tabela 6 . A desagregação apresentada é para um número de 13 setores, e as previsões são apresentadas em valores (milhões de tep) e em variação percentual ano a ano.

Os resultados para as previsões em milhões de tep em ambos os cenários permitem, primeiramente, identificar três grupos de setores segundo o porte da utilização de energia:

a) elevado consumo de energia: transportes, alimentos + bebidas e siderurgia;

b) moderado consumo de energia: agropecuária, papel e celulose, química e comércio + serviços;

c) baixo consumo de energia: extrativa mineral, minerais não-metálicos, não-ferrosos + outros metais, têxtil e o conjunto formado por outros setores.

Em termos agregados, no cenário expansionista o consumo total dos setores apresenta um vigoroso crescimento, saindo de 157,8 milhões de tep em 2005 para 174,3 milhões de tep em 2010, representando um aumento de 10,5\% entre esses dois anos, com uma média anual de crescimento de $2,5 \%$ ao ano. No cenário retraído, o consumo agregado também apresenta crescimento, mas de forma moderada, aumentando de 157,8 milhões de tep em 2005 para 160,9 milhões de tep em 2010, um aumento de apenas $2 \%$ entre esses dois anos.

Em termos desagregados, o cenário expansionista apresenta naturalmente taxas de crescimento mais vigorosas e todas positivas. Essas taxas variam de um mínimo de 1,1 \% (Têxtil em 2010) a um máximo de 4,9\% (Administração Pública em 20062010). No cenário retraído, ocorrem muitas taxas negativas de crescimento, em particular nos anos de 2009 e 2010 para todos os setores. 


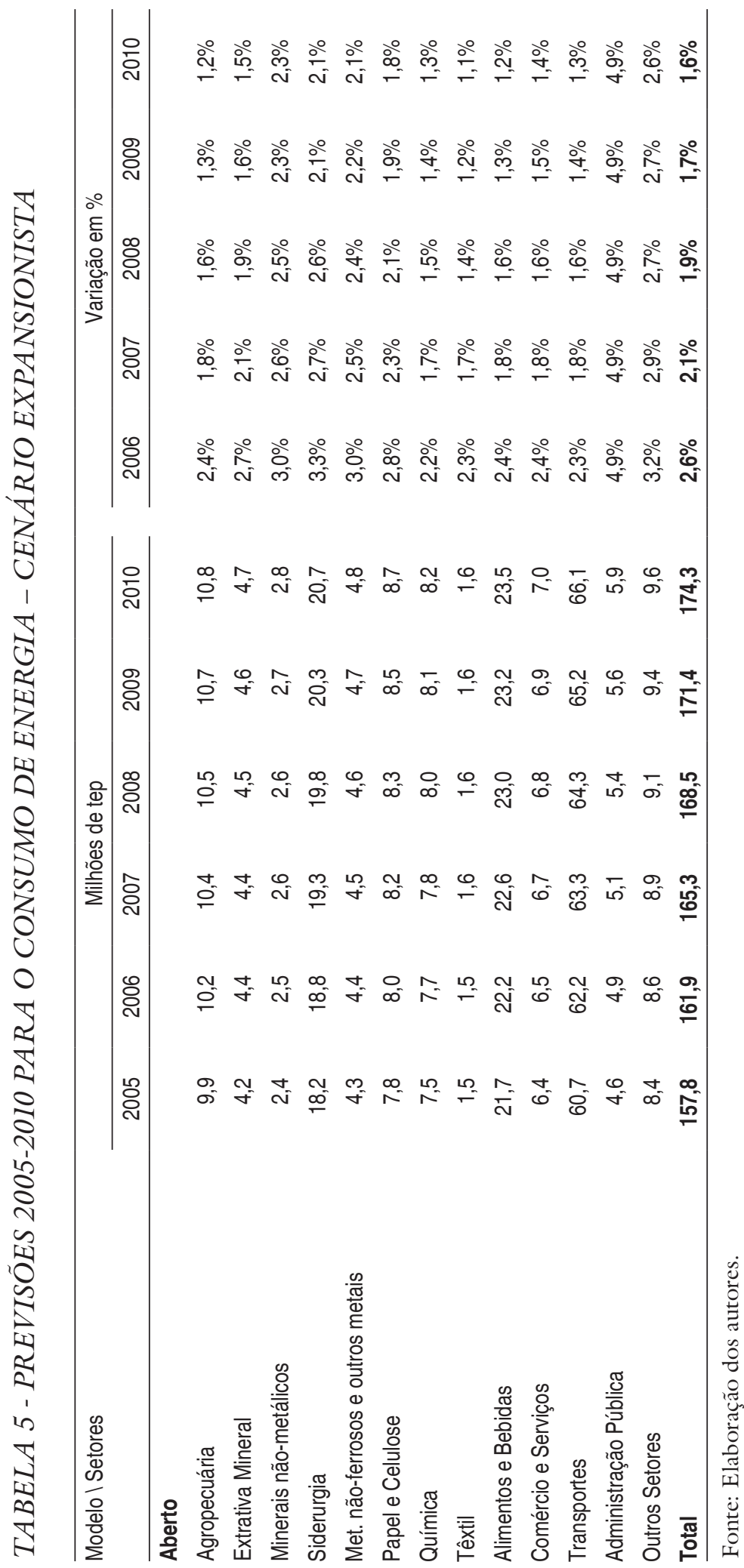




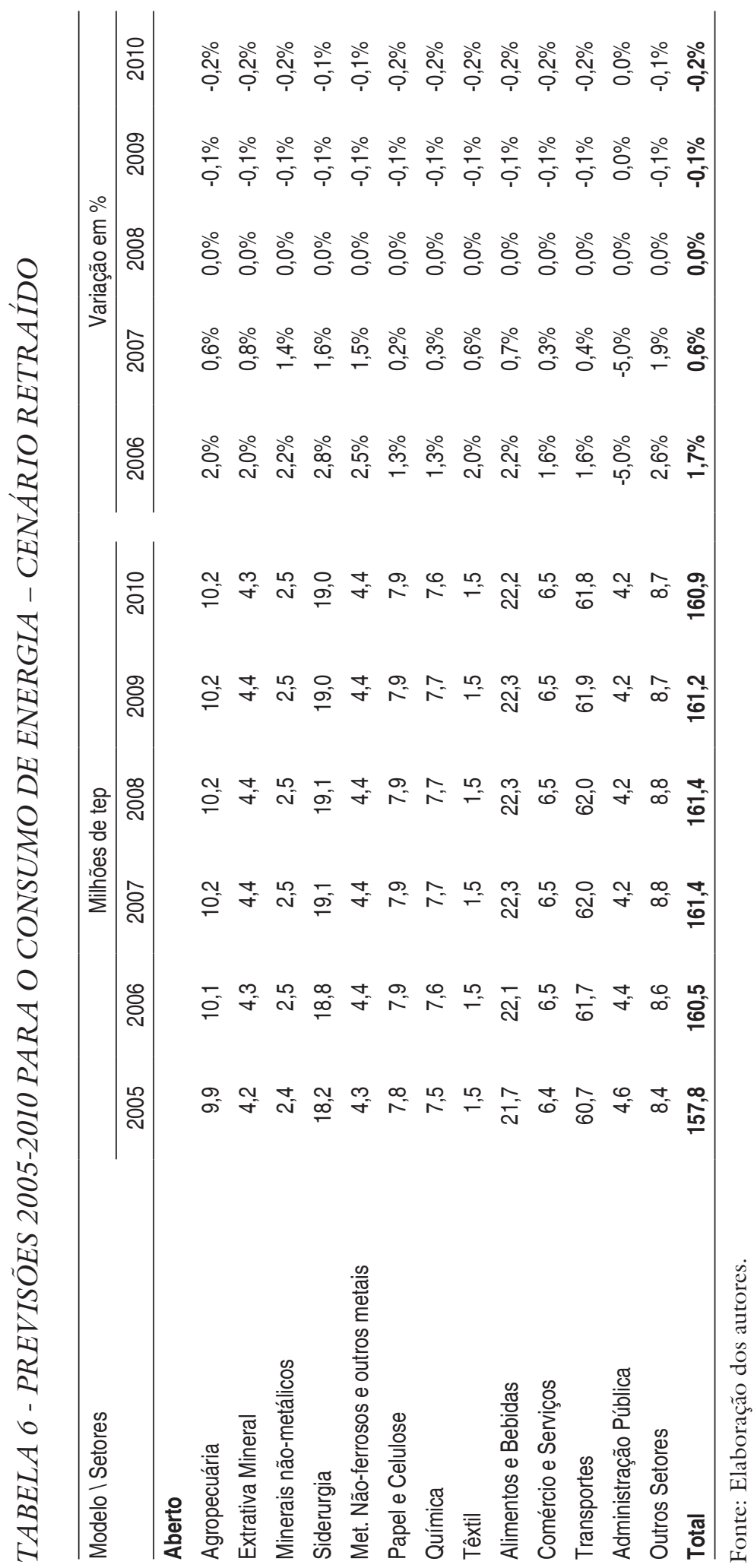




\section{DISCUSSÃO}

Conforme explicado na seção 4, o consumo total dos 13 setores não iguala o consumo final energético do Balanço Energético Nacional (BEN; ver Brasil/MME, 2004). Isso acontece porque no processo de compatibilização com a matriz de insumoproduto nacional, alguns ajustes precisaram ser feitos. Por exemplo, o consumo energético inclui também o consumo residencial, além do consumo total dos 13 setores da matriz de insumo-produto usada no modelo (ver seção 4).

Dados do BEN indicam que o consumo final energético em 2003 foi de 168,5 milhôes de tep, de forma que o consumo total dos 13 setores, de 131,8 milhões de tep naquele ano, representou $78,2 \%$. As previsões do modelo EC+IP aberto no cenário expansionista apontam que o consumo total dos setores ultrapassará a partir de 2009 o consumo final energético de 2003. A previsão feita é de 171,4 milhões de tep em 2009 e de 174,3 milhões de tep em 2010. No cenário retraído, o consumo final energético de 2003 não chega a ser ultrapassado em momento algum.

Considerando que atualmente o cenário expansionista talvez seja o mais provável, isso reforça a necessidade de que várias ações continuem sendo feitas pelos órgãos de planejamento energético para que oferta e demanda de energia se ajustem a médio e longo prazo. Uma vantagem de modelos desagregados como o aqui apresentado é que eles ajudam a identificar setores onde concentrar o foco de certas açóes. Por exemplo, o setor siderúrgico apresenta maior taxa de consumo de eletricidade dentre os 13 setores (MME, 2004) e, neste caso, as pesquisas de aumento da eficiência no uso de eletricidade são preponderantes. No caso de comércio e serviços, e também administração pública, outras ações como campanhas de conservação são mais relevantes.

O setor elétrico em 2003 forneceu eletricidade no montante de 29,4 milhões de tep, o que representava então cerca de $14,5 \%$ do consumo final energético. Desses 29,4 milhões de tep, cerca de $71 \%$, ou 21 milhões de tep, eram usados pelos 13 setores aqui considerados. Isto significa que $20,4 \%$ do consumo dos setores era de eletricidade. Embora o setor elétrico conte com um planejamento bastante elaborado e consubstanciado no Novo Modelo do Setor Elétrico deslanchado em 2004, segundo Furtado (2005), a perspectiva é de que o fornecimento só esteja garantido até 2009 . A se manter uma taxa próxima de $20,4 \%$ (de 2003) no âmbito de um cenário expansionista para 2005-2010, isso significa que o consumo de eletricidade dos setores chegaria a 37,4 milhões de tep em 2010 , um aumento de $27,3 \%$ sobre o consumo de 2003. 
Aqui, reforça-se a necessidade de gestões, dentro das regras do Novo Modelo, para garantir os novos investimentos em geração que tanto se fazem necessários. Vale observar que, ainda dentro das novas regras, o uso de modelos de previsão de longo prazo como o apresentado na seção anterior é relevante também para reduzir o risco dos agentes nos fechamentos de contratos de longo prazo acordados no âmbito da Câmara de Comercialização de Energia, dispositivo que pode viabilizar os investimentos privados de geração de energia nova (FURTADO, 2005).

\section{CONCLUSÃO}

Este trabalho apresentou um método alternativo, baseado em modelos integrados econométrico+insumo-produto, ou EC+IP, para se fazer previsões a longo prazo da demanda de energia no Brasil. Os resultados apresentados confirmam as preocupaçôes vigentes no debate energético de que um cenário de retomada do crescimento econômico pode ser restringido por um desequilíbrio negativo entre oferta e demanda de energia.

Na construção do modelo EC+IP, buscou-se fazer uso na montagem do módulo EC dos modernos enfoques da econometria de séries temporais, como as representações de modelos VCE e VAR. São dignas de nota as relações estabelecidas aqui, no âmbito de uma estratégia particular de integração por ligação, entre ausência/presença de co-integração das variáveis endógenas no modelo econométrico e o tipo de modelo IP (aberto/fechado) que deve ser usado. O esforço feito indica boas perspectivas para se avançar metodologicamente na integração dos modelos econométricos de séries temporais com os modelos de insumo-produto.

Como o modelo integrado que foi aqui apresentado é uma versão ainda simples, isso faz com que vários aspectos possam ser explorados para incrementá-lo em pesquisas futuras. Em particular, é relevante explorar o uso de mais variáveis exógenas no módulo EC uma vez que já existe atualmente uma significativa literatura para servir de base. Também, é preciso explorar a desagregação das previsões por tipos de energia consumida (petróleo, eletricidade etc.) e por região espacial de consumo. No último caso, isso envolverá o uso de modelos IP inter-regionais. Esses esforços de desagregação poderão trazer benefícios adicionais significativos para o desenho de políticas energéticas e a gestão estratégica do suprimento de energia. 


\section{REFERENCIAS}

AZZONI, C. R.; KADOTA, D. K. An econometric input-output model for the state of São Paulo. Brazil. (Texto para Discussão Nemesis.S/D). Disponível em: < http:// www.nemesis.org.br/>. Acesso em: 7 abr. 2005.

BRASIL. Ministério das Minas e Energia (MME). Brazilian energy balance 2002. Brasília, 2002. . Balanço Energético Nacional 2004. Brasília, 2004.

CONWAY, R. S. The Washington projection and simulation model: a regional interindustry econometric model. International Regional Science Review 13, p. $141-165,1990$.

ENDERS, W. Applied econometric time series. Nova York: Wiley, 2003.

FURTADO, C. Promessas e incertezas. Desafios do desenvolvimento. PNUD/IPEA, v. 2, n. 11, p. 16-23, 2005.

GUILHOTO, J. J. M.; SESSO FILHO, U A. Estimação da matriz insumo-produto a partir de dados preliminares das contas nacionais. Revista Economia Aplicada, v. 9, n.2, 2005 .

IPLANCE. Impactos da restrição de consumo de energia elétrica sobre a economia cearense. Governo do Estado do Ceará, Secretaria de Planejamento e Coordenação. Fundação Instituto de Pesquisa e Informação do Ceará - IPLANCE. Fortaleza, 2001.

ISRAILEVICH, P. R. et al. Forecasting structural change with a regional econometric input-output model. Journal of Regional Science, 37, p. 565-590, 1997.

ISRAILEVICH, P. R.; MAHIDHARA, R.; HEWINGS, G. J. D. The choice of input-output table embedded in regional econometric input-output models. CES 94-1, 1994.

IZARD, W. et al. Methods of regional analysis. Cambridge, MA: M.I.T. Press, 1960. . Methods of interregional and regional analysis. Brookfield: Ashgate, 1998.

LESAGE, J. P.; REY, S. J. Restrictions integrated econometric+input-output modeling. (Discussion Paper), 1994.

MILLER, R. E.; BLAIR, P. D. Input-output analysis. Foundations and extensions. Nova Jersey: Prentice Hall, 1985.

PROJECT LINK RESEARCH CENTRE. World Economic Outlook (meeting forecasts). Disponível em: <http://www.ipeadata.gov.br/ipeaweb.dll/NSerie> . Acesso em: 10 jun. 2005.

REY, S. J. Integrated regional econometric+input-output modeling: issues and opportunities. Papers in Regional Science, v. 79, p. 271-292, 2000.

; West, G. R.; Janikas, M. V. Uncertainty in integrated regional models. Economic Systems Research, v. 16, n. 3, p. 259-277, 2004. 